To the Editors:

\title{
Outcome after revascularisation of marginally viable limbs and dead limbs following lower limb arterial injuries
}

\author{
J Arudchelvam \\ Ceylon Medical Journal 2017; 62: 203-204 \\ DOI: http://doi.org/10.4038/cmj.v62i3.8526
}

Lower limb vascular injury occurs at a rate of $2 \%$ to $3 \%$ in civilian trauma [1]. Traditionally if the limb is anaesthetic and paralytic (dead limb) or after fasciotomy if two or more lower limb compartments are non-viable (marginally viable limb), revascularisation is not attempted because of the risks of reperfusion effects [2]. We have revascularised four dead or marginally viable limbs at our centre and the outcomes are presented below.

This is an analysis of prospectively maintained case records. Patients who presented with an arterial injury to teaching hospital Anuradhapura (THA) either with a viable limb (Group 1) or a dead or marginally viable limb (Group 2), from January 2015 to January 2016 were included in the analysis. Patients' demographic details, ischaemic time (time from the injury to revascularisation), artery injured, cause of arterial injury and the type of intervention were recorded. Comparisons between Groups 1 and 2 were made. Patients with mangled limbs, those systemically unfit for an intervention or incomplete records were excluded. Patients with an injury beyond the popliteal division were excluded from the analysis because of the difficulty in determining the number of vessels injured and these injuries were often associated with severe soft tissue injury. All patients underwent fasciotomy to confirm the viability. In Group 2 patients, revascularisation was attempted if they were young and otherwise systemically well and there were no severe soft tissue injury or local infection. All patients underwent revascularisation by a single surgeon. Post-operatively patients were closely monitored for reperfusion effects and sepsis.

Thirteen patients were included in the analysis (9 in Group 1 and 4 in Group 2). There were nine males and four females. The main cause of arterial injury in Group 1 was iatrogenic $(6 / 9,66.7 \%)$ whereas in Group 2 it was road traffic accident $(75 \%)$. In Group 1 four $(44.4 \%)$ were blunt injuries whereas in Group 2 , all injuries were blunt. Commonest artery injured in both Groups was the popliteal artery (four in Group, three in Group 2). The Mean ischaemic time in Group 1 was 11.3 hours (range 0.5 to 48 ) and in Group 2 it was 15.8 (range 7.5 to 25.5) hours. However this difference was not significant $(p=0.58)$. The mean time between presentation to hospital and revascularisation was 2.1 hours. All patients in Group 2 reported improved sensation at mean follow up of 23.0 weeks. However only one patient $(25 \%)$ had improvement of motor function. One patient had knee joint stabilisation and is walking now and other three patients can walk with difficulty (due to knee joint stiffness).

Consultant Vascular and Transplant Surgeon, Teaching Hospital Anuradhapura, Sri Lanka.

Correspondence: JA, E-mail: <joelaru@yahoo.com>. Received 29 March 2016 and revised version accepted 5 June 2017. 
This is a retrospective review of case notes. Commonest artery injured in both groups was the popliteal artery. All the injuries in Group 2 were blunt. In this series there are six iatrogenic injuries in Group 1 (66.7 \%) which is unusual. This included three infants who had accidental puncture and subsequent thrombosis of femoral arteries while attempting central venous line insertion and two patients who had iliac artery injury during spinal surgery.

Non-viability of a limb and risk of reperfusion is directly proportional to the duration of ischaemia. It is interesting to note that a limb which had been ischaemic for 25.5 hours in Group 2 was salvaged (Table 1). Hence the ischaemic time alone should not be considered as the deciding factor for revascularisation.

Traditionally, following an arterial injury, revascularisation was not attempted if the limb was dead or marginally viable because of the risk of reperfusion effect [2]. In our series such patients had only mild reperfusion effects like transient hemodynamic instability and electrolyte abnormalities and they recovered well. All patients in Group 2 had improvement in sensation and a quarter of patients had improvement of motor functions. This is an acceptable outcome, because most of the time non revascularisation results in amputation. But when revascularising dead limbs, all measures should be taken both pre and post operatively including close post-operative monitoring to prevent and treat reperfusion effects. If there is no response to interventions the limb should be immediately amputated to save the life of the patient. Therefore revascularisation should be attempted when the patient is systemically fit and the limb is not severely injured and there is no local infection.

\section{Conflicts of interest}

There are no conflicts of interest.

\section{Reference}

1. Compton C, Rhee R: Peripheral vascular trauma. Perspect Vasc Surg Endovascr Ther 2005; 17: 297-307.

2. Hafez HM, et al. Lower extremity arterial injury: Results of 550 cases and review of risk factors associated with limb loss. J Vasc Surg $2001 ; 33: 1212-9$.

Table 1. Patients who presented with dead / marginally viable limbs (Group 2)

\begin{tabular}{cccccccc}
\hline No & Age & Sex & Artery injured & Cause & $\begin{array}{c}\text { Type of } \\
\text { injury }\end{array}$ & $\begin{array}{c}\text { Ischemic time } \\
\text { (hours) }\end{array}$ & Procedure \\
\hline 1 & 60 & Male & Popliteal & Trap gun & blunt & 7.5 & Thrombectomy \\
2 & 25 & Male & Femoral & RTA & Blunt & 18 & RSVG Repair \\
3 & 48 & Female & Popliteal & RTA & Blunt & 12 & RSVG Repair \\
4 & & & & & & & RSVG Repair
\end{tabular}

RTA - Road Traffic Accident, RSVG - Reversed Saphenous Vein Graft

This is an open-access article distributed under the terms of the Creative Commons Attribution License, which permits unrestricted use, distribution, and reproduction in any medium, provided the original author and source are credited. 